\title{
FINITE ELEMENT METHODS FOR THE SIMULATION OF THIXOTROPIC FLOW PROBLEMS
}

\author{
N. Begum, A. Ouazzi and S. Turek \\ Institute for Applied Mathematics, LS III, TU Dortmund University, D-44227 Dortmund, \\ Germany \\ Naheed.Begum@math.tu-dortmund.de \\ Abderrahim.Ouazzi@math.tu-dortmund.de \\ ture@featflow.de (S. Turek)
}

Key words: Thixotropic Flows, Finite Element Methods (FEM), Newton-Multigrid, Monolithic, Generalized Navier-Stokes equations, Incompressible fluids

\begin{abstract}
This note is concerned with the application of Finite Element Methods (FEM) and Newton-Multigrid solvers for the simulation of thixotropic flow problems.

The thixotropy phenomena are introduced into viscoplastic material by taking into account the internal material micro structure using a scalar structure parameter. Firstly, the viscoplastic stress is modified to include the thixotropic stress dependent on the structure parameter. Secondly, an evolution equation for the structure parameter is introduced to induce the time-dependent process of competition between the destruction (breakdown) and the construction (buildup) inhabited in the material. Substantially, this is done simply by introducing a structure-parameter-dependent viscosity into the rheological model for yield stress material. The modified thixotropic viscoplastic stress w.r.t. the structure parameter is integrated in quasi-Newtonian manner into the generalized Navier-Stokes equations and the evolution equation for the structure parameter constitutes the main core of full set of modeling equations, which are creditable as the privilege answer to incorporate thixotropy phenomena. A fully coupled monolithic finite element approach has been exercised which manages the material internal micro structure parameter, velocity, and pressure fields simultaneously. The nonlinearity of the corresponding problem, related to the dependency of the diffusive stress on the material parameters and the nonlinear structure parameter models on the other hand, is treated with generalized Newton's method w.r.t. the Jacobian's singularities having a global convergence property. The linearized systems inside the outer Newton loops form a typical saddle-point problem which is solved using a geometrical multigrid method with a Vanka-like smoother taking into account a stable FEM approximation pair for velocity and pressure with discontinuous linear pressure and biquadratic velocity spaces. We examine the accuracy, robustness and efficiency of the Newton-Multigrid FEM solver throughout the solution of thixotropic viscoplastic flow problems in Couette device and in 4:1 contraction.
\end{abstract}




\section{Introduction}

We consider thixotropic viscoplastic (TVP) constitutive law based on Bingham model

$$
\left\{\begin{aligned}
\boldsymbol{\sigma}=2 \eta \mathbf{D}(\boldsymbol{u})+\tau \frac{\mathbf{D}(\boldsymbol{u})}{\|\mathbf{D}(\boldsymbol{u})\|}, & \text { if }\|\mathbf{D}(\boldsymbol{u})\| \neq 0 \\
\|\boldsymbol{\sigma}\| \leq \tau, & \text { if }\|\mathbf{D}(\boldsymbol{u})\|=0
\end{aligned}\right.
$$

where $\mathbf{D}(\boldsymbol{u})$ denotes the strain rate tensor. The norm for a tensor $\Lambda$ is given by $\|\Lambda\|=$ $\sqrt{\operatorname{Tr}\left(\Lambda^{2}\right)}$. We use $\|\mathbf{D}(\boldsymbol{u})\|$ and $\|\mathbf{D}\|$ alternately. $\eta$ denotes plastic viscosity, and $\tau$ defines a yield stress that is a threshold parameter from which the material start yielding. The shear stress has two contributions: a viscous part, and a strain rate independent part. Thixotropic phenomena, as a competition of two processes, build up and breakdown, are introduced into rheological model for yield stress material through a structure-parameter. A class of models for plastic viscosity and yield stress w.r.t. sturcure parameter is given in the table 1. The initial plastic viscosity and yield stress are denoted by $\eta_{0}$ and $\tau_{0}$,

Table 1: Some relations between rheological parameters and the structure parameter
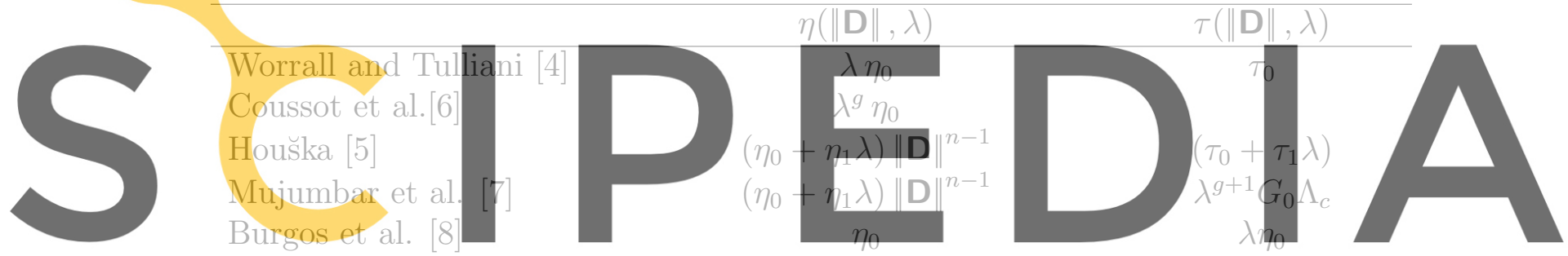

Dullaert \& Mewis [9]

$\lambda \eta_{0}$

$\lambda G_{0}(\lambda\|\mathbf{D}\|) \Lambda_{c}$

Register for free at https//www.scipedia.com to download the version without the watermark

respectively, in the absence of any thixotropic phenomena. $\eta_{1}$ and $\tau_{1}$ represent the rates

of changes of plastic viscosity and yield stress, respectively, w.r.t. structure parameter. $\Lambda_{c}$ is the critical elastic strain (either constant or variable) and $G_{0}$ is the elastic modulus of unyielded material. The time-dependent process of competition between the destruction (breakdown) and the construction (buildup) inhabited in the material is given by the following structure-parameter equation

$$
\left(\frac{\partial}{\partial t}+u \cdot \nabla\right) \lambda=\mathcal{F}-\mathcal{G}
$$

where, $\mathcal{F}$ and $\mathcal{G}$ are two nonlinear functions for buildup and breakdown. The table 2 shows different thixotropic models. $a$ and $b$ are rate constants for creation (buildup) and breakage (breakdown), and $g, p, m, n$ are rate indices.

The quasi-Newtonian modeling approach for thixotropic flows is based on a viscosity approach for yield stress flows in which an extended viscosity is introduced $\mu(\cdot, \cdot)$ dependent on the strain rate and the structure parameter. Indeed, let us introduce the 
Table 2: Some expressions for buildup and breakdown functions of structure evolution equation

\begin{tabular}{lcc} 
& $\mathcal{F}$ & $\mathcal{G}$ \\
\hline Worrall and Tulliani [4] & $a(1-\lambda)\|\mathbf{D}\|$ & $b \lambda\|\mathbf{D}\|$ \\
Coussot et al. [6] & $a$ & $b \lambda\|\mathbf{D}\|$ \\
Houška [5] & $a(1-\lambda)$ & $b \lambda^{m}\|\mathbf{D}\|$ \\
Mujumbar et al.[7] & $b \lambda\|\mathbf{D}\|$ \\
Burgos et al. [8] & $a(1-\lambda)$ & $b \lambda\|\mathbf{D}\| \exp (g\|\mathbf{D}\|)$ \\
Dullaert \& Mewis $[9]$ & $b \lambda\|\mathbf{D}\| t^{-p}$ \\
\hline
\end{tabular}

second invariant of the strain rate tensor $D_{\mathrm{II}}=\frac{1}{2}(\mathbf{D}(\boldsymbol{u}): \mathbf{D}(\boldsymbol{u}))$, and define the regularized viscosity, as for instance [14]:

$$
\mu\left(D_{\mathbb{I}}, \lambda\right)=\eta\left(D_{\mathbb{I}}, \lambda\right)+\tau\left(D_{\mathbb{I}}, \lambda\right) \frac{\sqrt{2}}{2} \frac{1}{\sqrt{D_{\mathbb{I}}}}\left(1-e^{-k \sqrt{D_{\mathbb{I}}}}\right)
$$

A general system of the governing equations in primitive variables $\boldsymbol{u}, p$, and $\lambda$ for the flow of thixotropic material is described as follows:
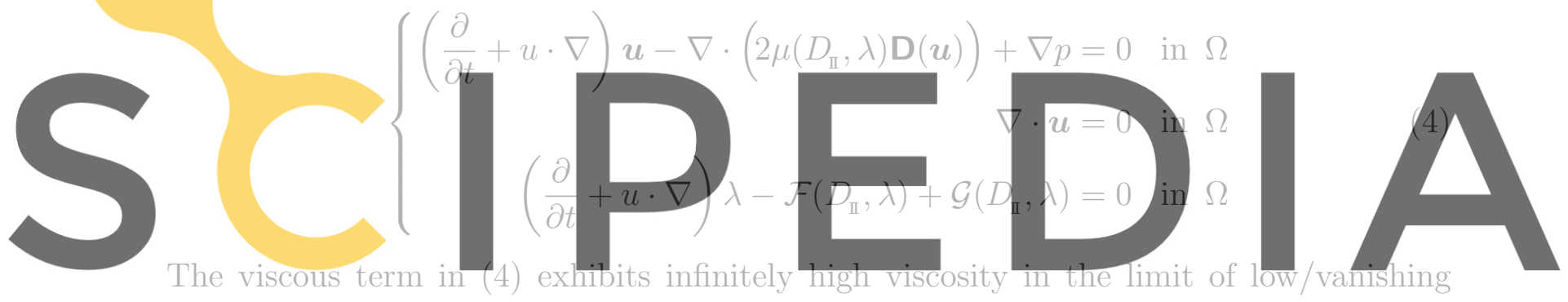

The viscous term in (4) exhibits infinitely nign viscosity in the limit on low/vanishing Register for free at:ibttps/4wwy. scipedia.com to download the version withput the watermark to be very high which deteriorates the solver on the other hand.

\section{Finite element discretization}

To derive the variational form for thixotropic flows, we consider the spaces $\mathbb{T}:=$ $L^{2}(\Omega), \mathbb{V}:=\left(H_{0}^{2}(\Omega)\right)^{2}$, and $\mathbb{Q}:=L_{0}^{2}(\Omega)$ associated, respectively, with the corresponding $L^{2}$-norm, $\|\cdot\|_{0}, H^{1}$-norm, $\|\cdot\|_{1}$, and $L^{2}$-norm, $\|\cdot\|_{0}$. Let $\tilde{\boldsymbol{u}}:=(\lambda, \boldsymbol{u}, p) \in\left(\mathbb{T} \cap H^{1}(\Omega)\right) \times \mathbb{V} \times \mathbb{Q}$, and $\tilde{\boldsymbol{v}}:=(\xi, \boldsymbol{v}, q) \in \mathbb{T} \times \mathbb{V} \times \mathbb{Q}$ be a test function. The weak formulation for the thixotropic flows reads: Find $\tilde{\boldsymbol{u}} \in\left(\mathbb{T} \cap H^{1}(\Omega)\right) \times \mathbb{V} \times \mathbb{Q}$ s.t.

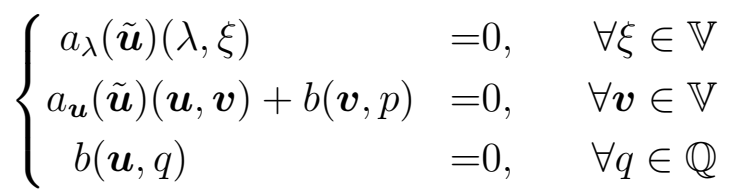

Or, equivalently: Find $\tilde{\boldsymbol{u}} \in\left(\mathbb{T} \cap H^{1}(\Omega)\right) \times \mathbb{V} \times \mathbb{Q}$ s.t.

$$
a_{\lambda}(\tilde{\boldsymbol{u}})(\lambda, \xi)+a_{\boldsymbol{u}}(\tilde{\boldsymbol{u}})(\boldsymbol{u}, \boldsymbol{v})+b(\boldsymbol{v}, p)-b(\boldsymbol{u}, q)=0 \quad \forall \tilde{\boldsymbol{v}} \in \mathbb{T} \times \mathbb{V} \times \mathbb{Q}
$$


where $a_{\lambda}(\tilde{\boldsymbol{u}})(\cdot, \cdot), a_{\boldsymbol{u}}(\tilde{\boldsymbol{u}})(\cdot, \cdot)$, and $b(\cdot, \cdot)$ are given as follows

$$
\begin{gathered}
a_{\lambda}(\tilde{\boldsymbol{u}})(\lambda, \xi)=\int_{\Omega}\left(-\mathcal{F}\left(D_{\mathrm{II}}, \lambda\right)+\mathcal{G}\left(D_{\mathrm{II}}, \lambda\right)\right) \xi d \Omega+\int_{\Omega} \boldsymbol{u} \cdot \nabla \lambda \xi d \Omega \\
a_{\boldsymbol{u}}(\tilde{\boldsymbol{u}})(\boldsymbol{u}, \boldsymbol{v})=\int_{\Omega} 2 \mu\left(D_{\mathrm{II}}, \lambda\right) \mathbf{D}(\boldsymbol{u}): \mathbf{D}(\boldsymbol{v}) d \Omega+\int_{\Omega} \boldsymbol{u} \cdot \nabla \boldsymbol{u} \boldsymbol{v} d \Omega \\
b(\boldsymbol{v}, q)=-\int_{\Omega} \nabla \cdot \boldsymbol{v} q d \Omega .
\end{gathered}
$$

The finite element approximations of the problem (6) have to take care of its saddle point character, due to the bilinear form (9). Furthermore, since thixotropic flows are usually slow, the only remaining issue is the control/continuity of the bilinear form (7) in the norm of space $\mathbb{T}$.

We opt for the higher order stable pair biquadratic for velocity and piecewise linear discontinuous for the pressure, $Q_{2} / P_{1}^{\text {disc }}$, and higher order quadratic for structure parameter $Q_{2}$ with the appropriate stabilization terms $[12,13]$. Indeed, let the domain $\Omega$ be partitioned by a grid $K \in \mathcal{T}_{h}$ which are assumed to be open quadrilaterals such that $\Omega=\operatorname{int}\left(\bigcup_{k \in \mathcal{T}_{h}} \bar{K}\right)$. For an element $K \in \mathcal{T}_{h}$, we denote by $\mathcal{E}(K)$ the set of all 1 dimensional edges of $K$. Let $\mathcal{E}_{i}:=\bigcup_{k \in \mathcal{T}_{h}} \mathcal{E}(K)$ be the set of all interior element edges of

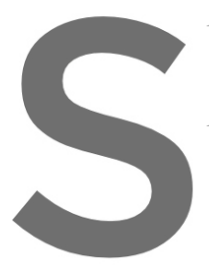
the grid $\mathcal{T}_{h}$.
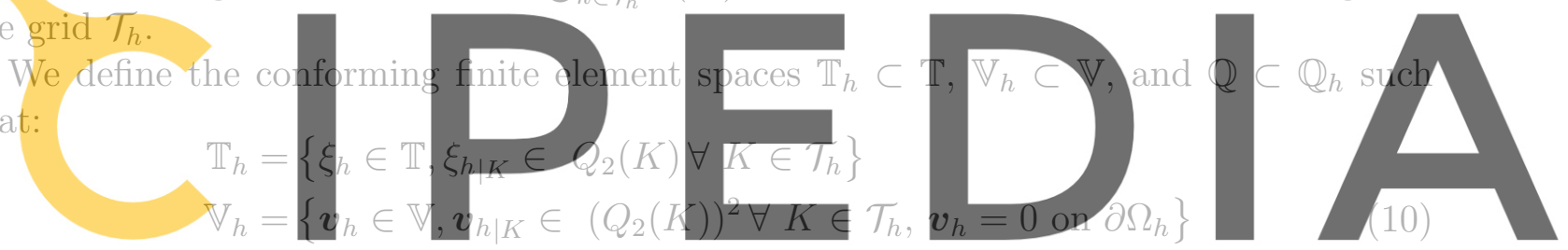

\section{$\mathbb{Q}_{h}=\left\{q_{h} \in \mathbb{Q}, q_{h \mid K} \in P_{1}^{\text {disc }}(K) \forall K \in \mathcal{T}_{h}\right\}$}

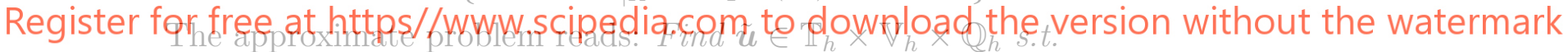

$$
\left\{\begin{aligned}
a_{\lambda}(\tilde{u})(\lambda, \xi)+j_{\lambda}(\lambda, \xi) & =0, & & \forall \xi \in \mathbb{T}_{h} \\
a_{\boldsymbol{u}}(\tilde{\boldsymbol{u}})(\boldsymbol{u}, \boldsymbol{v})+b(\boldsymbol{v}, p) & =0, & & \forall \boldsymbol{v} \in \mathbb{V}_{h} \\
b(\boldsymbol{u}, q) & =0, & & \forall q \in \mathbb{Q}_{h}
\end{aligned}\right.
$$

Or, equivalently: Find $\tilde{\boldsymbol{u}} \in \mathbb{T}_{h} \times \mathbb{V}_{h} \times \mathbb{Q}_{h}$ s.t.

$$
a_{\lambda}(\tilde{\boldsymbol{u}})(\lambda, \xi)+j_{\lambda}(\lambda, \xi)+a_{\boldsymbol{u}}(\tilde{\boldsymbol{u}})(\boldsymbol{u}, \boldsymbol{v})+b(\boldsymbol{v}, p)-b(\boldsymbol{u}, q)=0, \quad \forall \tilde{\boldsymbol{v}} \in \mathbb{T}_{h} \times \mathbb{V}_{h} \times \mathbb{Q}_{h}
$$

where the stabilization term $j_{\lambda}(\cdot, \cdot)$ is given as follows

$$
j_{\lambda}(\lambda, \xi)=\sum_{E \in \mathcal{E}_{i}} \gamma_{\lambda}|E| \int_{E}[\nabla \lambda][\nabla \xi] d \sigma
$$

The stabilization (13) is consistent and makes the coercivity and continuity match in $\mathbb{T}_{h}$ associated with the norm $\|\cdot\|$, where

$$
\|\xi\|^{2}=\|\xi\|_{0}^{2}+j_{\lambda}(\xi, \xi)
$$




\section{Generalized discrete Newton}

We use the Newton method to approximate the nonlinear residuals. Let $\mathcal{R}(\tilde{\boldsymbol{u}})=$ $\left(\mathcal{R}_{\lambda}(\tilde{\boldsymbol{u}}), \mathcal{R}_{\boldsymbol{u}}(\tilde{\boldsymbol{u}}), \mathcal{R}_{p}(\tilde{\boldsymbol{u}})\right)=\left(\mathcal{R}_{(\lambda, \boldsymbol{u})}(\tilde{\boldsymbol{u}}), \mathcal{R}_{p}(\tilde{\boldsymbol{u}})\right)$ denote the residuals for the system (12). The nonlinear iteration is updated with the correction $\delta \tilde{\boldsymbol{u}}, \tilde{\boldsymbol{u}}^{k+1}=\tilde{\boldsymbol{u}}^{k}+\delta \tilde{\boldsymbol{u}}$. Then, the Newton linearization gives the following approximation for the residuals:

$$
\begin{aligned}
\mathcal{R}\left(\tilde{\boldsymbol{u}}^{l+1}\right) & =\mathcal{R}\left(\tilde{\boldsymbol{u}}^{l}+\delta \tilde{\boldsymbol{u}}\right) \\
& \simeq \mathcal{R}\left(\tilde{\boldsymbol{u}}^{l}\right)+\left[\frac{\partial \mathcal{R}\left(\tilde{\boldsymbol{u}}^{l}\right)}{\partial \tilde{\boldsymbol{u}}}\right] \delta \tilde{\boldsymbol{u}}
\end{aligned}
$$

The Newton's method iterations, assuming invertible Jacobians, are given as follows

$$
\tilde{u}^{l+1}=\tilde{u}^{l}-\omega_{l}\left[\frac{\partial \mathcal{R}\left(\tilde{u}^{l}\right)}{\partial \tilde{u}}\right]^{-1} \mathcal{R}\left(\tilde{u}^{l}\right)
$$

The damping parameter $\omega_{l} \in(0,1)$ is chosen such that

$$
\left\|\mathcal{R}\left(\tilde{u}^{l+1}\right)\right\| \leq\left\|\mathcal{R}\left(\tilde{u}^{l}\right)\right\|
$$
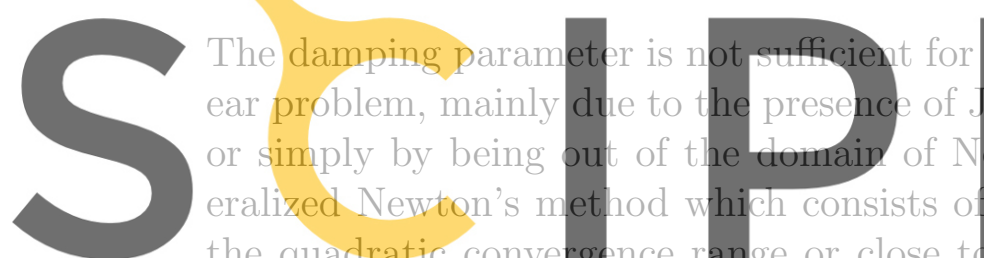

the quadratic converg
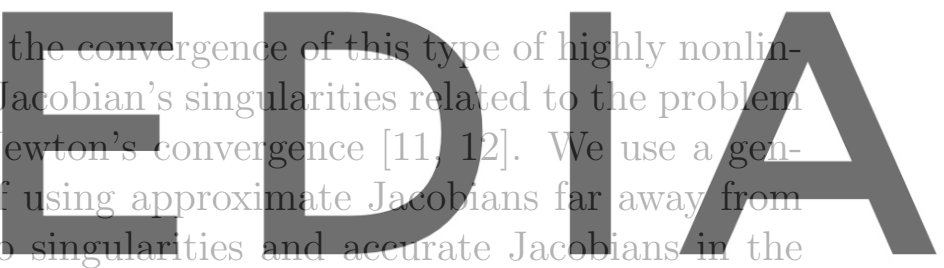

quadratic region of convergence in an adaptive way $[2,11]$. Indeed, based on a priori

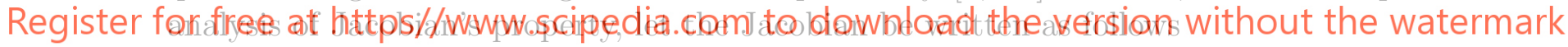

$$
\left(\frac{\partial \mathcal{R}\left(\tilde{\boldsymbol{u}}^{l}\right)}{\partial \tilde{\boldsymbol{u}}}\right)=\left(\frac{\partial \tilde{\mathcal{R}}\left(\tilde{\boldsymbol{u}}^{l}\right)}{\partial \tilde{\boldsymbol{u}}}\right)+\delta_{l}\left(\frac{\partial \hat{\mathcal{R}}\left(\tilde{\boldsymbol{u}}^{l}\right)}{\partial \tilde{\boldsymbol{u}}}\right)
$$

The Jacobian is splitted to a direct sum of corresponding operators with different properties. The parameter $\delta_{l} \in(0,1)$ is solely dependent on the rate of actual residual convergence [11]. It is worth mentioning that the operator-related damped Jacobian method (17) is related to the continuous Newton's method. Furthermore, for the analysis of adaptive discrete Newton's method where the Jacobian is evaluated using the divided difference we refer to [2]. The Jacobian approximation is only dependent on the rate of the actual residual convergence $\left(\left\|\mathcal{R}^{l}\right\| /\left\|\mathcal{R}^{l-1}\right\|\right)$. This generalized Newton's method assures a global nonlinear convergence $[2,11]$. 


\section{Monolithic multigrid linear solver}

To develop an appropriate linear solver, we segregate the Jacobian as follows

$$
\left(\frac{\partial \mathcal{R}(\tilde{\boldsymbol{u}})}{\partial \tilde{\boldsymbol{u}}}\right)=\left(\begin{array}{cc}
\frac{\partial \mathcal{R}_{(\lambda, \boldsymbol{u})}(\tilde{\boldsymbol{u}})}{\partial(\lambda, \boldsymbol{u})} & \frac{\partial \mathcal{R}_{\boldsymbol{u}}(\tilde{\boldsymbol{u}})}{\partial p} \\
\frac{\partial \mathcal{R}_{p}(\tilde{\boldsymbol{u}})}{\partial \boldsymbol{u}} & 0
\end{array}\right)
$$

which is a saddle point problem. Then, the resulting linear system is treated with a Multilevel Pressure Schur Complement (MPSC) approach with Vanka-like smoother, i.e.

$$
\tilde{u}^{k+1}=\tilde{u}^{k}-\omega_{k} \sum_{K \in \mathcal{T}_{h}}\left(\left(\frac{\partial \mathcal{R}\left(\tilde{\boldsymbol{u}}^{l}\right)}{\partial \tilde{u}}\right)_{\mid K}\right)^{-1} \mathcal{R}\left(\tilde{u}^{l}\right) \mid K
$$

In (19), we solve exactly on real element, $K$, and perform an outer Gauß-Seidel iteration

$[3,17]$. We use standard geometric multigrid solver for linearized system with standard $Q_{2}$ and $P_{1}^{\text {disc }}$ restriction and prolongation operators.

The combination of a stable finite element approximations, $Q_{2} / P_{1}^{\text {disc }}$, for Stokes problem together with multigriel results in a high nunnerically acc multigrid solver.

5
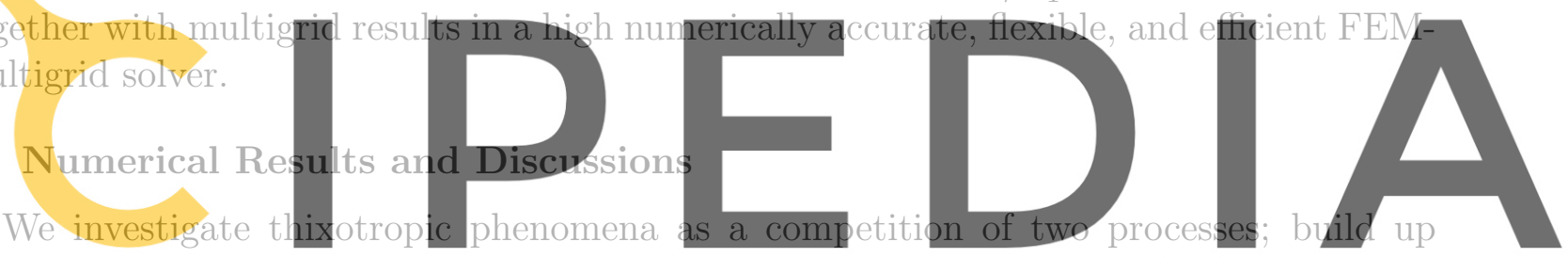

and breakdown. From archetypical thixotropic models given in Table (1-2), we employ

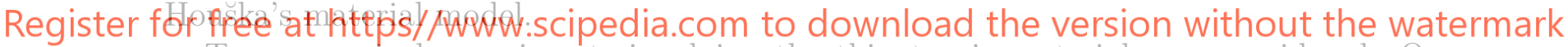

Two numerical experiments involving the thixotropic materials are considered: Our

first test is related to the simulation of thixotropic material in Couette device. Here, we examine the liquid-/solid transitions of shear-localization and shear-banding in terms of the thixotropy breakdown parameter. Our second numerical experiment pertains to entry flow of thixotropic material in 4:1 contraction. Here, we investigate the shape and extent of unyielded zones in terms of thixotropic yield stress parameter.

Clearly, tracking the true unyielded/yielded zone in any flow configuration is regularization dependent and requires relatively large values for $k$ to accurately capture the yielded/unyielded regions. It is worth emphasizing here that our solver is so robust that the solutions with big values of the regularization parameter are possible in the flow situations where material departures from being simple visocplastic and approaches to thixo-viscoplastic. However, the analysis of solver statistics requires more investigations which go beyond the scope of this short note. The choice of regularization parameter in viscosity function is $k=10^{4}$, since the solutions remain intact for further increment in $k$ [1]. 


\subsection{Thixotropic flows in Couette device}

For numerical solutions for thixotropic flows in Couette device, we consider two concentric cylinders with an inner and an outer radii $r_{\text {in }}$ and $r_{\text {out }}$ defined as $r_{\text {in }}=\zeta /(1-\zeta)$ and $r_{\text {out }}=1 /(1-\zeta)$; where $\zeta$ denotes the radii ratio. The inner cylinder is made under rotation speed, $\boldsymbol{u}=u\left(r_{\text {in }}\right) e_{\theta} ; u\left(r_{\text {in }}\right)=1$ RPM, while the outer cylinder is kept static, $r_{\text {out }}=0$ RPM $[10,15]$.

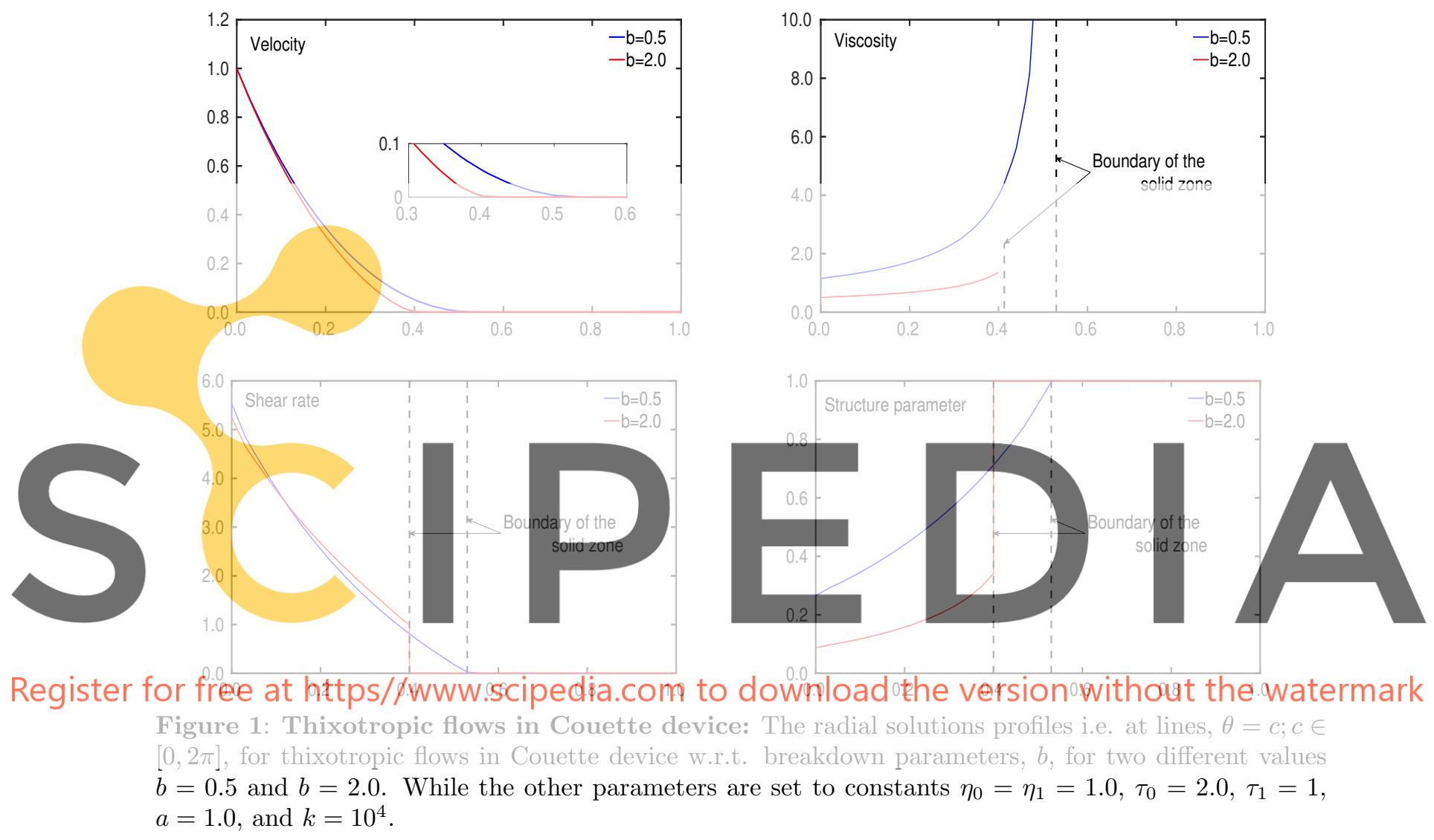

Figure 1 shows the radial solutions profiles, velocity, structure parameter, shear rate, and viscosity w.r.t. breakdown parameter. A smooth transition from the flowing regimes to static ones is given with a small values of the breakdown parameter. The model allows for very sharp transitions as well for higher breakdown parameter. The structure parameter which controls the buildup and the breakdown regions matches perfectly with the flowing and plug zones indicated by the shear rate. Furthermore, the velocity on one hand and the shear rate and structure parameter on the other hand come into contact perfectly in the transition points for both "localization" (lower breakdown parameter, $b=0.5$ ) and "shear banding" (higher breakdown parameter $b=2.0$ ). The viscosity shows a discontinuity jump to infinity which is specific to thixotropic yield stress rather than the continuously diverging as for a simple yield stress [16]. 


\subsection{Thixotropic flows in contractions}

In this section, we analyse the simulations of thixo-viscoplastic flows in a 4:1 curved contraction. Information on the considered computational domain is displayed in Figure 2. The fully-developed flow conditions according to Houška thixotropic model are imposed at entry and exit together with no-slip on the top and bottom walls of reservoir and downstream channel. Our emphasis is to examine the impact of thixotropic yield stress on morphology of unyielded zones, which plays a key role for preserving the main rheological characteristics of materials with yield stress. Furthermore, we investigate the transitions in shape and extent of the flowing/rigid zones w.r.t breakdown parameter on isobands of material micro-structuring level $\lambda$.

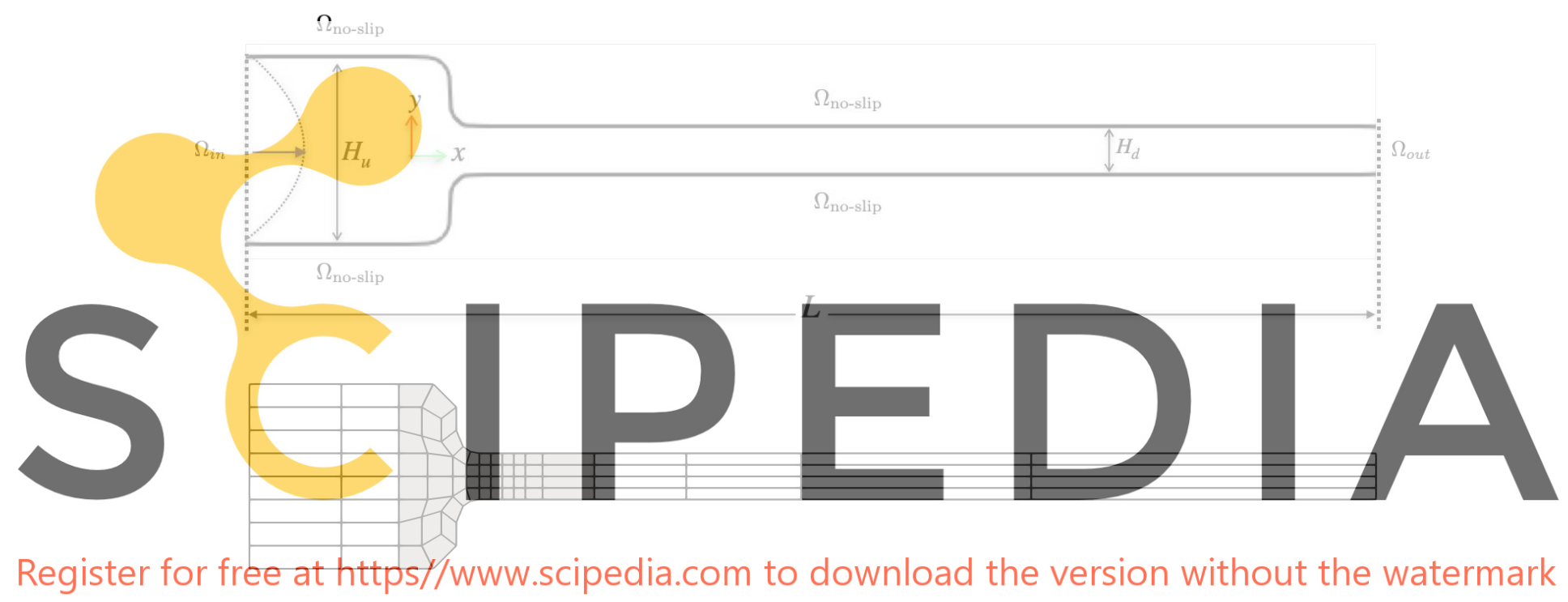

Figure 2: 4:1 Contraction domain and the corresponding coarse mesh

In Figure 3, we present progressive escalation of solid (unyielded) regions in flow domain as a function of thixotropic yield-stress parameter. The solid (unyielded) zones are distinguished from yielded ones by shading in Figure 3. Three distinct unyielded regions are formed inside 4to1 enclosure filled by thixo-viscoplastic material: $(a)$ rigid zone close to the inlet containing an envelope of stagnant/unyielded material, $(b)$ unyielded dead spaces near the corners of reservoir, and $(c)$ an unyielded region containing (nearly) stagnant material which occupies the zone formed by the cross-section change, henceforth called downstream channel unyielded region. It is observed that thixotropic plasticity has quite strong effect to give rise to the thickness of envelope of the solid zones, which are much bigger in the reservoir than the downstream channel, and this trend is readily understood due to relatively slow flow (hence stress level) in the reservoir. Moreover, the unyielded regions near the corner also stretches in the axial direction by increasing 
yield-stress intensity. Consequently, for extreme values of $\tau_{1}$ only the large gradient area close to the corners of entrance zone remain yielded.

In Figure 4, we present the spatial distribution of material structuring level to illustrate the impact of thixotropy breakdown parameter $b$. It is worth-mentioning here that pipeline design involves the pipe sections with contractions/expansions shapes, for instance pipeline fittings. A better understanding of material inherent thixotropy helps in circumventing the flow restarts issues, and allows for a cost-friendly equipment designs. It is clear from Figure 4, that increasing the breakdown parameter induces more beakdown layers close to the walls of downstream channel preventing the material from rest inside long pipelines.

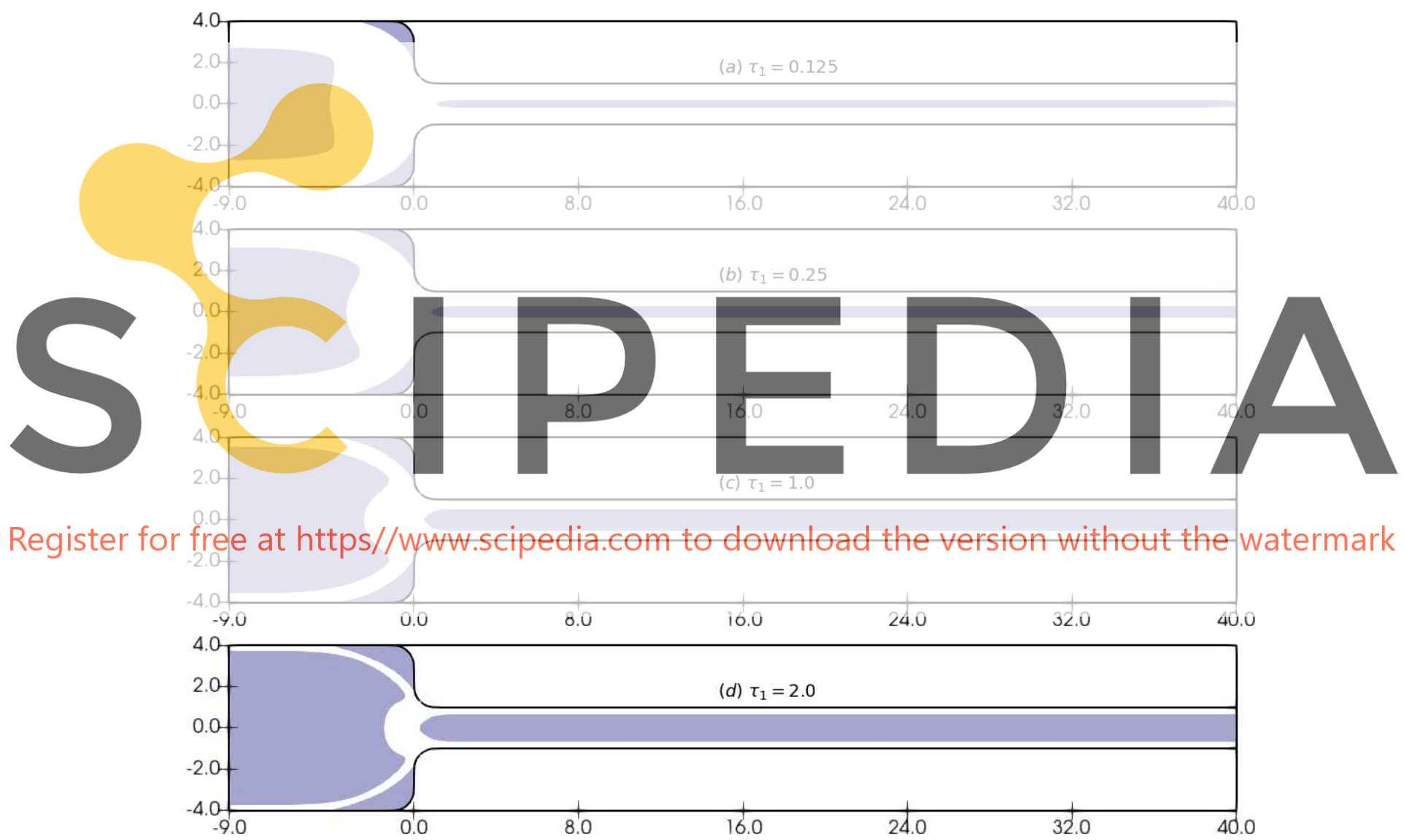

Figure 3: Thixotropic flows in contractions: The progressive growth of unyielded regions as a function thixotropic yield stress parameter $\tau_{1}$ for thixotropic flow $\mathrm{s}$ in 4:1 contractions. While the other parameters are set to constants $\eta_{0}=\eta_{1}=1.0, \tau_{0}=0.0, a=b=1.0$, and $k=10^{4}$. 
(i) Upstream channel

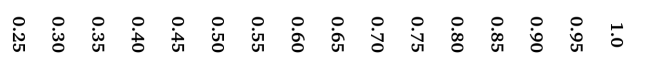
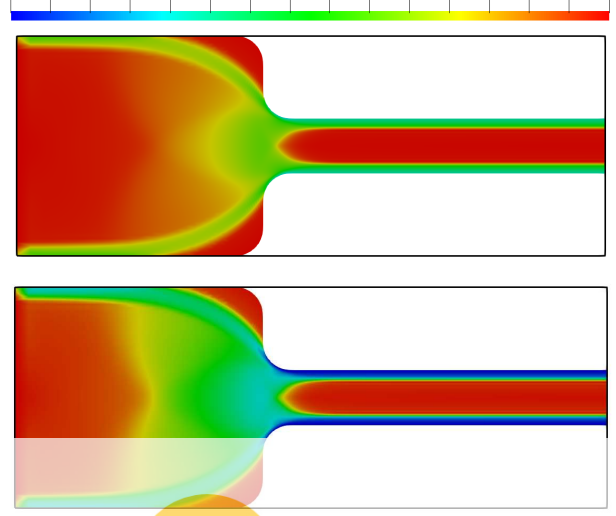

(ii) Downstream channel
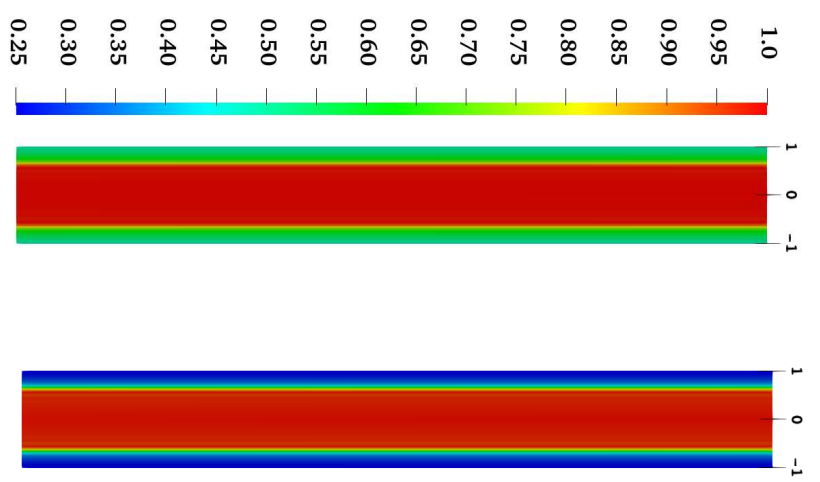

Figure 4: Thixotropic flows in contractions: The structuring level of material $\lambda$ for thixotropic flows in 4:1 contractions w.r.t. breakdown parameters, $b$, for two different values $b=1.0$ (Top) and $b=2.0$ (Bottom). While the other parameters are set to constants $\eta_{0}=\eta_{1}=1.0, \tau_{0}=0.0, a=1.0, \tau_{1}=2.0$, and $k=10^{4}$.

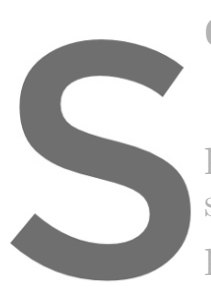

6 Summary

We presented a

roach for thixotropic

stable $Q_{2} / P_{1}^{\text {disc }} \mathrm{FE}$ app

proximation for the strention

combination of a stable finite element appre
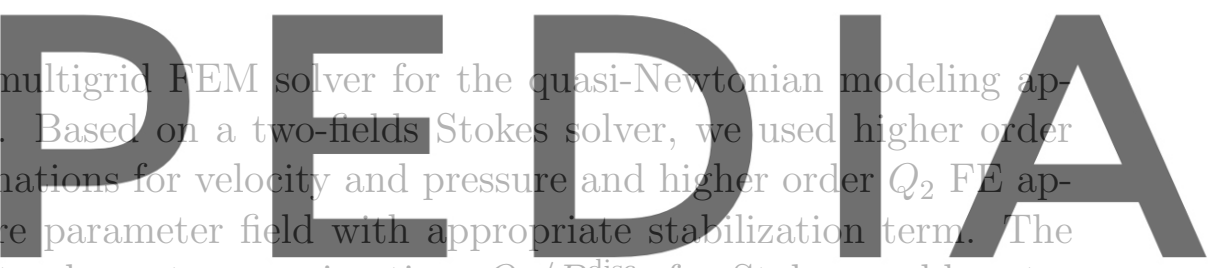

-approximation, $Q_{2} / P_{1}^{\text {disc }}$, for Stokes problem to-

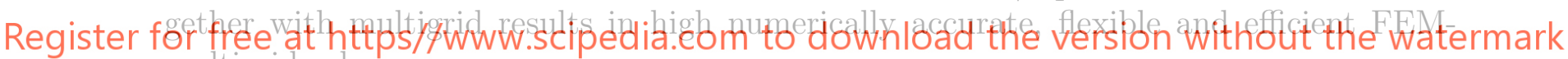
multigrid solver.

The quasi-Newtonian model for thixotropic flows is highly nonlinear due to the dependency of the extended viscosity on the material parameters (shear rate and structureparameter) and the regularization parameter. The nonlinearity is handled with generalized Newton's method w.r.t. the Jacobian's singularities having a global convergence property.

We analyzed the application of using the quasi-Newtonian modeling approach for thixotropic flows, and the accuracy, robustness and efficiency of the Newton-Multigrid FEM solver throughout the solution of the thixotropic flows using two configurations of thixotropic flows in Couette device and contraction domains.

Very high values for regularization are required to achieve accurate solutions. Fortunately, the solver is so robust that the solutions are obtained for higher values for regularization parameter. Consequently, some very important characteristics of thixotropic flows were captured with the quasi-Newtonian thixotropic model; as for instance, localization, shear banding, and discontinuous jump to infinity for viscosity. 


\section{Future outlook}

It is interesting to observe that unyielded zones in upstream and downstream parts of contraction domain do not merge at all. Physically, this happens because the unyielded material in the vicinity of center of reservoir becomes more rigid due to its inelastic nature. That means, the material has no capability to deform elastically. Thus, when the material elements cross the contraction zone, they are not able to undergo even a small scale elastic crosswise extension and remain disconnected and travel like a rigidbody in downstream channel. Further investigation is to consider the nonzero flow below the critical yield stress limit. In our current settings of employed model, the rheological manifestation of thixotropy is limited to viscoplastic materials. Therefore, we anchor the future modification of constitutive law towards thixotropic elasto-viscoplastic (TEVP) model that could accommodate both elastic and plastic characteristics of material in single backbone.

\section{Acknowledgement}

This work was supported by Deutscher Akademischer Austauschdienst (DAAD) through the funding program /-ID: Research Grants-Doctoral Programmes in Germany 2016/17- (57214224). The authors also acknowledge the support by LS3 and LiDO3 team at ITMC, TU Dortmund University.

\section{REFERENCES}

[1] Ouazzi, A., Begum, N., and Turek, S. Newton-multigrid FEM solver for the simulation of quasi-newtonian modeling of thixotropic flows. Ergebnisberichte des Instituts für Angewandte Mathematik Nummer 638, Fakultät für Mathematik, TU Dortmund University 638, (2021).

[2] Fatima, A., Turek, S., and Ouazzi, A. An adaptive discrete Newton method for regularization-free Bingham model. Ergebnisberichte des Instituts für Angewandte Mathematik Nummer 635, Fakultät für Mathematik, TU Dortmund University 635, (2021)

[3] Damanik, H., Hron, J., Ouazzi, A., and Turek, S. A monolithic FEM-multigrid solver for non-isothermal incompressible flow on general meshes. J. Comp. Phys. (2009) 228:38693881.

[4] Worrall, W. E., and Tuliani, S. Viscosity changes during the ageing of clay-water suspensions. Trans. Brit. Ceramic Soc. (1964) 63:167-185.

[5] Houška, M. Engineering aspects of the rheology of thixotropic liquids. PhD thesis, Faculty of Mechanical Engineering, Czech Technical University of Prague, (1981).

[6] Coussot, P., Nguyen, Q. D., Huynh, H. T., and Bonn, D. Viscosity bifurcation in thixotropic, yielding fluids. J. Rheol. (2002) 46(3):573-589. 
[7] Mujumdar, A., Beris, A. N., and Metzner, A. B. Transient phenomena in thixotropic systems. J. Nonnewton. Fluid Mech. (2002) 102(2):157-178.

[8] Burgos, G. R., Alexandrou, A. N., and Entov, V. Thixotropic rheology of semisolid metal suspensions. J. Mater. Process. Technol. (2001) 110(2):164-176.

[9] Dullaert, K., and Mewis, J. A Structural kinetics model for thixotropy. J. Nonnewton. Fluid Mech. (2006) 139:21-30.

[10] Jenny, M., Kiesgen de Richter, S., Louvet, N., Skali-Lami, S., and Dossmann, Y. TaylorCouette instability in thixotropic yield stress fluids. Phys. Rev. Fluids (2017) 2:023302023323.

[11] Mandal, S., Ouazzi, A., and Turek, S. Modified Newton solver for yield stress fluids, Proceedings of ENUMATH 2015, the 11th European Conference on Numerical Mathematics and Advanced Applications, Springer,(2016):481-490

[12] Ouazzi, A. Finite Element Simulation of Nonlinear Fluids. Application to Granular Material and Powder, Shaker Verlag, Achen (2006).

[13] Turek, S., and Ouazzi, A. Unified edge-oriented stabilization of nonconforming FEM for incompressible flow problems: Numerical investigations. J. Numer. Math. (2007) 15(4):299322 .

[14] Papanastasiuo, T. C., Flow of materials with yield. J. Rheol., (1987) 31:385-404.

[15] Langroudi, M. K., Turek, S., Ouazzi, A., and Tardos, G. An investigation of frictional and collisional powder flows using a unified constitutive equation. Powder Technology, (2009) 197(1-2):91-101.

[16] Moller, P., Fall, A., Chikkadi, V., Derks, D., and Bonn, D. An attempt to categorize yield stress fluid behaviour. Phil Trans. R. Soc. A, (2009) 367:5139-5155.

[17] Turek, S. Efficient solvers for incompressible flow problems. Lecture Notes in Computational Science and Engineering, Springer, Berlin (1999). 